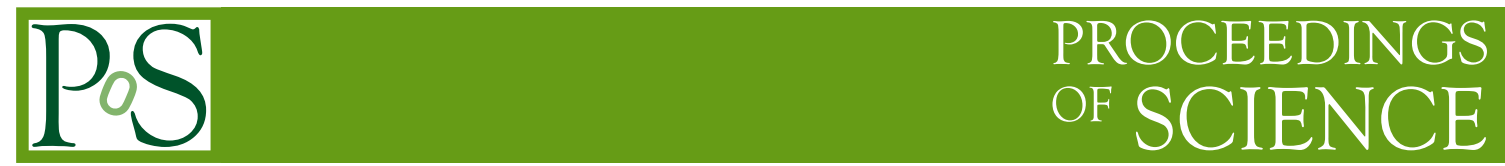

\title{
High Energy Astrophysical Neutrino Signals
}

\section{Todor Stanev*}

Bartol Research Institute and Department of Physics and Astronomy, University of Delaware, Newark, DE 19716, U.S.A.

E-mail: stanev@bartol.udel.edu

\begin{abstract}
The goal of this paper is to give a popular account for the high energy astrophysical neutrinos detected by the IceCube Collaboration. We start with a discussion of the importance of the detection of astrophysical neutrinos and of the atmospheric neutrino background for such detections. Then we discuss the high energy neutrino events. At the end of the presentation we compare the arrival directions of these events with those of extragalactic gamma-ray sources and ultrahigh energy cosmic rays.
\end{abstract}

Frontier Research in Astrophysics,

25-30 May 2014

Palermo, Italy

${ }^{*}$ Speaker. 


\section{Introduction}

Until a year ago we had no idea if we will ever detect astrophysical neutrinos and gain knowledge on the strength of such signals and on the shape of their energy spectrum. We always expected that there are many sources of astrophysical neutrinos. The most suspect galactic neutrino sources are the supernova remnants where we believe galactic cosmic rays are accelerated. Because of the very small neutrino interaction cross section we knew that high energy neutrinos may easily propagate to us from very large distances and thus may point at the extragalactic cosmic ray sources where ultrahigh energy cosmic rays (UHECR) are accelerated.

Since astrophysical neutrinos should be produced by high energy cosmic rays there have been many different models for their generation. The astrophysical neutrinos may be the result of hadronic interactions of the accelerated cosmic rays in the vicinity of acceleration sites if the matter density of their sites is significant. They could also be produced inside the accelerating astrophysical object in photoproduction interactions $p+\gamma \rightarrow p+\pi^{ \pm}+\ldots$ with the local photon field. A typical neutrino production model of this type is the one by Eli Waxman \& John Bahcall [1] that attempted to set an upper limit on the fluxes of astrophysical neutrinos based on the emissivity of UHECR in the Universe and on their acceleration spectrum. The source of UHECR and astrophysical neutrinos in this model are gamma ray bursts (GRB).

\subsection{Why do we care about astrophysical neutrinos}

Cosmic rays are charged nuclei and they are deflected in scattering in the magnetic fields of the Universe, galactic or extragalactic. For this reason we have to look at the neutral secondary particles generated in cosmic ray interactions close to their acceleration sites. The possible origin of the astrophysical neutrinos in powerful astrophysical objects is the main reason for the high interest in the directions they come from. Neutrinos are only generated in hadronic interactions of nuclei or $\gamma$-rays and they can prove what the importance of hadronic processes is in the dynamics of the astrophysical objects. We have a good sample of $\mathrm{TeV}$ gamma-rays, detected by the air Cherenkov telescopes HESS, Magic and Veritas for many of which the sources are determined and electronically published in the online gamma-ray catalog TeVCat [2]. Gamma rays, however, could be produced either in hadronic interactions where they come from pion and kaon decays, or by electrons that undergo inverse Compton scattering. In this way the detection of high energy $\gamma$-rays does not point at astrophysical objects where hadronic interactions happen. Only neutrinos certainly point at such objects. In addition, extragalactic gamma rays are absorbed in propagation in pair production interactions $\gamma \gamma \rightarrow e^{+} e^{-}$with the microwave background and other photon fields of the Universe, while the main energy loss of the neutrinos is due to the expansion of the Universe.

We know well that cosmic rays hitting the Earths atmosphere generate cascades that contain all kinds of secondary particles including neutrinos due to charged pions and kaons decays. These neutrinos have been detected in underground detectors. Neutrino oscillations were discovered by the SuperKamiokande detector by observations of atmospheric neutrinos. The biggest underground detector is IceCube [3] at the South Pole of the Earth. This is a $\mathrm{km}^{3}$ detector situated under 1450 meters of ice, which corresponds to a coverage with $1.35 \times 10^{5} \mathrm{~g} / \mathrm{cm}^{2}$. This way the IceCube detector is shielded by the high energy muons created in the atmosphere that need to be of energy exceeding $500 \mathrm{GeV}$ to penetrate the shielding. 
Being the biggest underground detector IceCube has measured the energy spectra of the atmospheric muon and electron neutrinos to the highest possible energy. These spectra, compared to a calculation of the atmospheric neutrino spectra are shown in the left hand panel of Fig. 1. The neutrino fluxes in this figure are in units of $E_{v} F_{v}$. There are couple of obvious conclusions that the viewer can draw from Fig 1:

1. The flux of atmospheric muon neutrinos and antineutrinos is significantly higher than this of electon neutrinos and antineutrinos.

2. The energy spectrum of the electron neutrinos is steeper than this of muon neutrinos.

The reason is that muon neutrinos are generated in charged meson decays and in the subsequent muon decays. The atmospheric electron neutrinos are mostly generated in muon decays. For this reason the energy spectrum of muon neutrinos is steeper by one power of the energy than those of the all nucleon cosmic ray spectrum. If it is approximately $E_{n}{ }^{-2.7}$ this of the atmospheric muon neutrinos is $E_{V}{ }^{-3.7}$. As the electron neutrino and antineutrino spectrum is the result of two subsequent decays it is steeper by two powers of the energy and should approximately be $E_{v}{ }^{-4.7}$. The real calculations of these spectra [4] estimate the spectra of these neutrinos much better taking into account the real shape of the cosmic rays spectrum and all processes involved in their production.
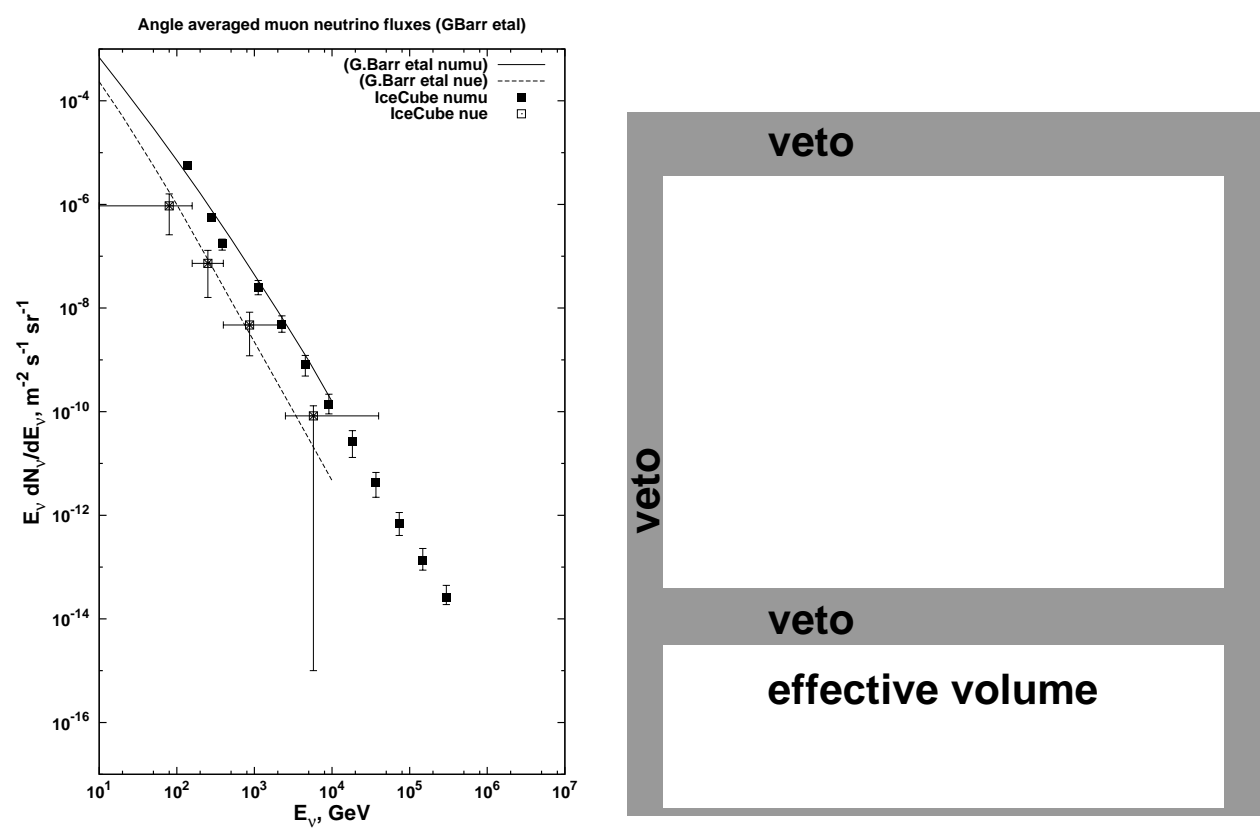

Figure 1: Lefthand panel: the measured fluxes of amospheric muon and electron neutrinos and antineutrinos; Righthand panel: Geometry of the IceCube high energy detection scheme of starting events.

\section{IceCube detection of astrophysical neutrinos}

It is obvious from Fig. 1 that the search for astrophysical neutrinos should start at high energy because the atmospheric neutrino background is too high at low energy. In the case of IceCube the discovery started in 2012, when the japanese group of IceCube members started looking for the 
highest energy events. The construction of IceCube was finished in December 2010 qand it now consists of 86 strings. Each string is $1 \mathrm{~km}$ long and contains 60 digital optical modules (DOMs) which contain the phototubes and related electronics. This analysis [5] looked at the data from the first year of the completed IceCube (May 2011 - May 2012) and from the previous year when IceCube consisted of 79 strings. The amount of data corresponds to 616 working days of the experiment. Since the detector was different during these two years different conditions on the total amount of photoelectrons were applied to the data. Two events were found with very high amount of detected photoelectrons, $7.0 \times 10^{4}$ and $9.6 \times 10^{4}$, which after comparison with MonteCarlo calculations correspond to 1.04 and $1.14 \mathrm{PeV}$ deposited in the detector. Both events look like cascades in the ice, i.e. do not contain muon tracks.

Te next step of analysis used the data from the same two years (with a slightly higher statistics of 662 days) and attempted to have the same sensitivity to events arriving from different directions. The geometry of this search is drawn in the right hand panel of Fig. 1. It looked at events starting inside the detector not including the veto regions. Since the atmospheric muon background is highest at the top of the detector the veto region on the top is thickest and on the bottom it is thinner. The veto also includes a region inside the detector where the ice is not very transparent and the sensitivity there is smaller.

This analysis revealed 28 events that deposited in the detector between 30 and 1,200 $\mathrm{TeV}$ of energy [6] versus a background of $10.6_{-3.6}^{+5.0}$ events coming from atmospheric muons and neutrinos. The events included track events consistent with muon neutrinos and cascade events similar in shape with the previously discovered PeV events. Examples of cascade (left hand panel) and track events (right hand panel) are shown in Fig. 2. Four of the track events start near to the top of the detector and point down, and are thus consistent with the atmospheric muons background of $6 \pm 3.4$ events. One of these events had hits in the IceTop surface air shower array IceTop, compatible in arrival time and direction. The two $\mathrm{PeV}$ events were also identified in this analysis.
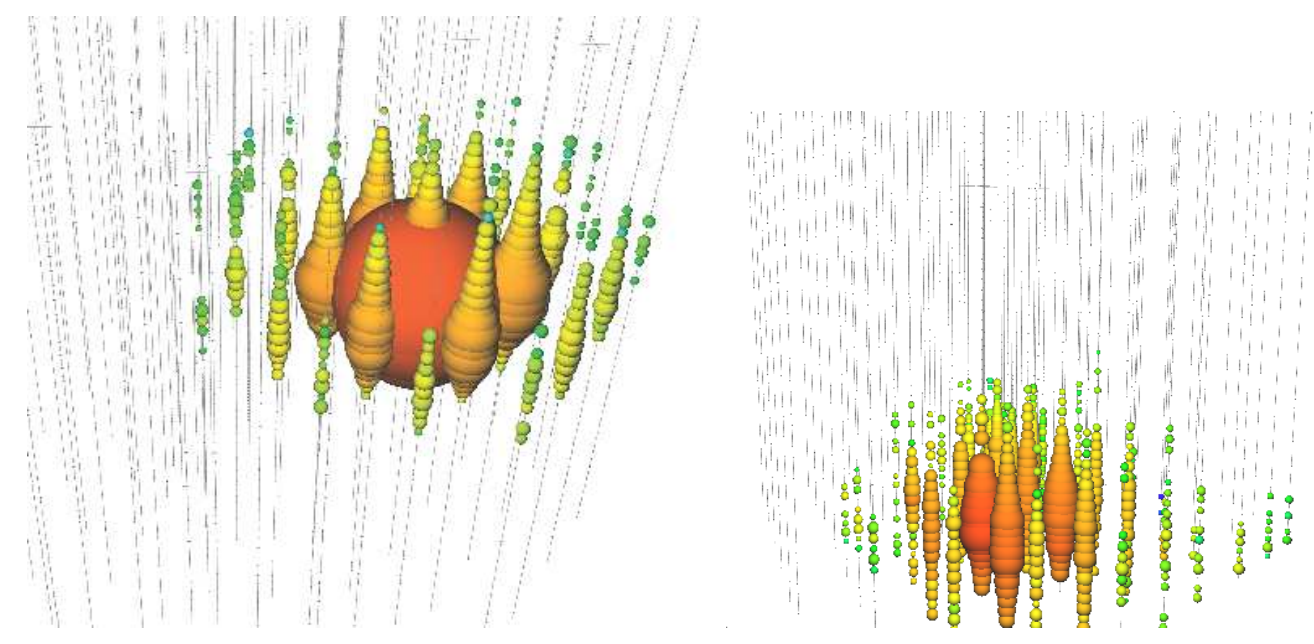

Figure 2: Lefthand panel: Cascade event depositing $1140 \mathrm{TeV}$ of energy in IceCube; Righthand panel: Muon track event depositing $82 \mathrm{TeV}$ of energy in IceCube. The muon neutrino interacts inside the detector and the resulting muon moves from left to right; Both events are extracted from the IceCube web page. 
These are the two types of high energy events, a large fraction of which must be astrophysical, that IceCube measured. The muon tracks must follow muon neutrinos or antineutrinos charged current (CC) interactions outside the detector. The cascade events are either electron neutrinos, tau neutrinos, or neutral current (NC) interactions of all neutrino flavors. Even at a PeV the tau meson decay length is small, of the order of the distance between two DOMs on a string and it is difficult to distinguish from electron neutrinos. Muon tracks allow the reconstruction of their direction within about $1.5^{\circ}$, but there is lots of uncertainty about the energy of the neutrino. For cascade events the energy estimate is good, because the whole event is contained inside IceCube, however the direction is not determined well. When an electron neutrino interacts inside IceCube, the results of this interaction look like an explosion and the photoelectrons seem to go out of a central point. Because of that the neutrino direction determination is worse than several degrees. For a couple of events the error band reaches $40^{\circ}$ around the central value. One should never forget that the energies determined by IceCube are not the ones of the neutrinos, they are the energies deposited by the neutrino interactions inside the detector.

Very recently IceCube released the data from the same analysis of one more year of observations [7], which revealed nine more high energy events.

There were a couple of surprises during the initial analysis of these events. Everybody who has thought or worked on neutrino detection believed that astrophysical neutrinos will be detected mostly as muon tracks entering IceCube from below. Because of this the appearance of 21 cascades and only seven muon tracks in the first sample was surprising. On the other hand the number of cascades could be estimated as the sum of the NC interactions of all three neutrino flavors and the CC interactions of $v_{e}$ and $v_{\tau} 3 \times \sigma_{N C}+2 \times \sigma_{C C}$ while the number of tracks is only proportional to $\sigma_{C C}$. If we take the NC cross section to be $1 / 2$ of the CC cross section (it is a bit less) the ratio of cascades to tracks should be 3.5, while in the sample it is three including all track events. The other surprise was that more events entered the detector from above (negative declination) rather than from below. A calculation of the effective area $A_{\text {eff }}$ of IceCube for upgoing and for downgoing neutrinos (also shown in the journal paper [6]) shows that $A_{\text {eff }}$ is higher for downgoing events. The reason is that high energy neutrinos going through the Earth are absorbed. The absorbtion depends on the pathlength of the neutrinos inside the Earth. The absorbtion is highest (and the absorption energy threshold is lowest) for vertically upwardgoing events. Having these two facts in mind both initial surprises are easily explained. The declination of the whole three years event sample is shown versus the deposited energy in the detector in Fig. 3. The error bars on the declination of individual events are the average errors of the reconstructed event directions.

\section{Arrival directions of the high energy neutrino events}

Although the available statistics of neutrino events that deposited more than $30 \mathrm{TeV}$ in IceCube is still very small and not all of the background events can be identified0, it is extremely interesting to compare the arrival directions of these neutrinos to other relevant signals. There are two types of such signals: $\mathrm{TeV} \gamma$-rays and ultrahigh energy cosmic rays. Many of the sources of $\mathrm{TeV}$ gamma rays have been identified as either galactic or extragalactic. All galactic $\gamma$-ray sources are very close to the galactic plane. The identified extragalactic sources, as well as the gamma-ray sources that are not identified, come almost isotropically from the fields of view of the $\gamma$-ray telescopes. 


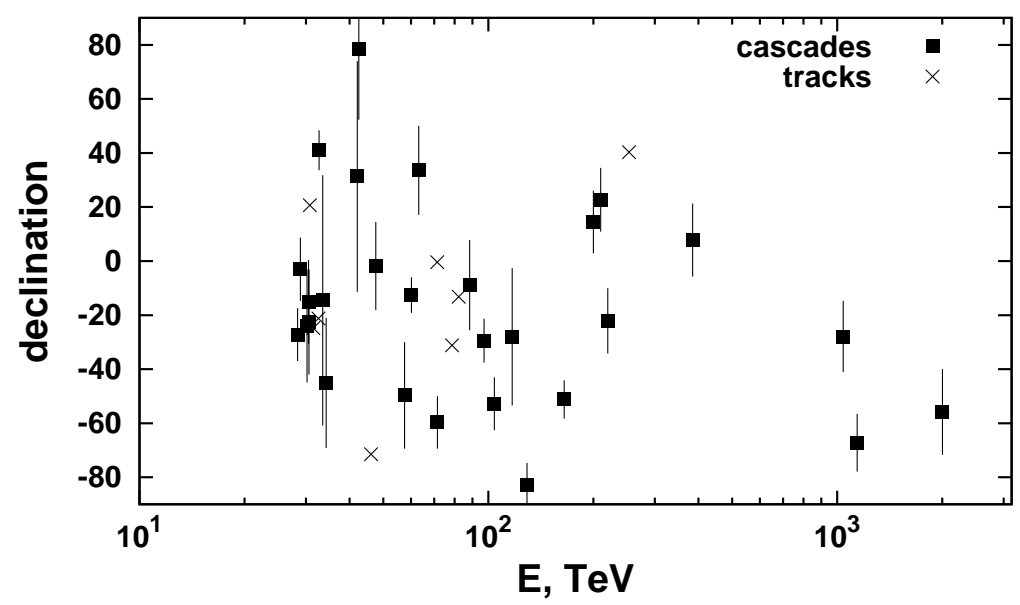

Figure 3: Declination versus deposited energy in the detector for all 37 high energy neutrino events (eight muon tracks) in IceCube.

The other relevant signals are the arrival directions of the highest energy cosmic rays detected by the Auger observatory, the High Resolution Fly's Eye (HiRes), and the Telescope Array (TA) detector. We think of these ultrahigh energy cosmic rays (UHECR) as particles accelerated by extragalactic sources since it is impossible to achieve energies exceeding $10^{19} \mathrm{eV}$ in the suspected galactic sources of cosmic ray acceleration. It is worth saying here that UHECR cannot be accelerated more than 50-100 Mpc away from our Galaxy while astrophysical neutrinos could come to us from much further away. The distances to the $\mathrm{TeV} \gamma$-ray sources are also limited because of gamma-ray absorption.

Although UHECR scatter in the magnetic fields, their scattering angles should be small and possibly reveal the region, if not the source, of their acceleration. Unfortunately the amount of scattering depends strongly on the chemical composition of these UHECR the results on which are not conclusive yet. HiRes and the Telescope Array interpretation of the longitudinal development of the air showers they create in the atmosphere is that the primary UHECR are very light, either Hydrogen or Helium nuclei. The Auger Observatory analysis reveals a chemical composition that becomes heavier above an energy of $10^{18} \mathrm{eV}$. Think about protons and Fe nuclei. The scattering angle of an iron nucleus is going to be 26 times higher than that of a proton with the same energy. It is still interesting to compare the arrival direction of these events.

Figure 4 shows the arrival directions of the IceCube high energy neutrino events with the directions of the extragalactic $\mathrm{TeV} \gamma$-ray sources and with the directions of the Auger, and the Telescope Array cosmic rays with energy above $57 \mathrm{EeV}\left(5.7 \times 10^{19} \mathrm{eV}\right)$. One should not forget that IceCube can see neutrinos coming from any direction of the sky (with some absorption inside the Earth) while $\mathrm{TeV} \gamma$-ray telescopes and air shower detectors only observe events within their field of view. When we combine the results from different detectors (three $\mathrm{TeV} \gamma$-ray detectors, or Auger and TA) the field of view becomes more uniform, but it still does not cover the whole sky.

There is no obvious anisotropy in any of the signals. Concentrating on the IceCube high energy neutrino events we may feel that in a couple of regions the signal density is higher, but 


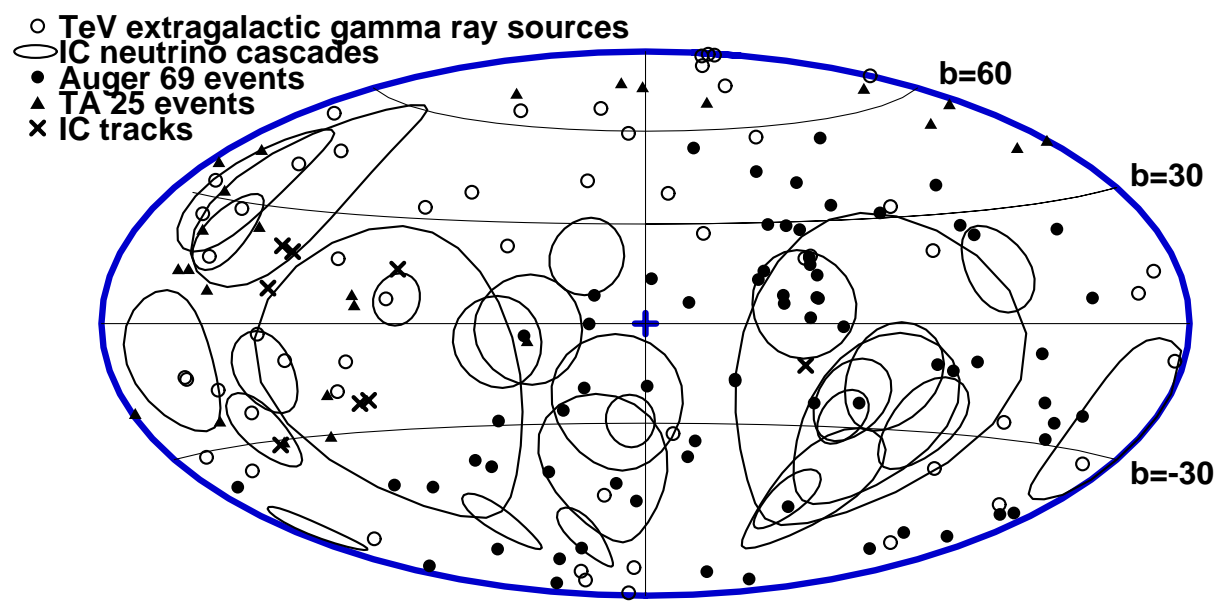

Figure 4: Arrival directions of different types of signals in galactic coordinates.

after a statistical analysis accounting for all errors, this has no significance. One of these regions is relatively close to the galactic center. Otherwise we do not see any tendency of event concentration around the galactic plane as we see with the confirmed galactic $\mathrm{TeV} \gamma$-ray sources. There are several cases where several UHECR are incide the error bars of an IceCube cascade, like in the case of Auger UHECR around Cen A but none of these cases have any statistical significance. I am afraid we will have to wait for a while to have more significant statistics than one shown in the graph before we can even suspect some common sources of the different types of events.

\section{Discussion}

The important question here is how long we have to wait. We suspect that the Auger Observatory has many more UHECR than the 69 events published three years ago. The same must be true for the Telescope Array. These two collaborations have started working together to determine better the degree of anisotropy of their events above $10^{19} \mathrm{eV}$. So we expect that relatively soon we will have more data on UHECR. In the case of $\mathrm{TeV} \gamma$-rays we do not expect a large increase of the statistics before the Cherenkov telescope array is built. The current $\mathrm{TeV} \gamma$-ray telescopes will continue finding new extragalactic sources but the strength of the signals is limited by the distance to them and I do not expect the number of such sources to increase rapidly.

What about high energy neutrinos? Currently IceCube is much bigger than the existing and even planned observatories in the sea, such as Antares and Km3Net, so that we cannot expect a significant increase of statistics. There are several groups in the IceCube Collaboration that are pursuing different analysis methods from the one described above and some of them will certainly find more events. A bigger increase of statistics could only come from a bigger new detector.

The IceCube Collaboration became excited when the high energy neutrino events were detected and there are now discussions of increasing the size of the detectors by a factor of five or more. This is possible if the distance between strings is significantly increased. The threshold for neutrino detection will increase (originally IceCube was designed for detection of $\mathrm{TeV}$ neutrinos) 
but the detection of high energy neutrino events will increase. The construction of such an extension, when it happens, will take some time. The detailed design itself will take a couple of years. The construction, although the collaboration is much better now than in the beginning, should also take several years and the new events will start coming in at higher rate after that.

There is also the development of new detection methods mostly based on the idea of Askaryan [8] about the radio emission from high energy cascades. There have already been several test experiments that have detected radio pulses of possible neutrino interactions and also from air showers. One of these experiments, ARA, is deployed at the edge of IceCube and attempts to detect high energy neutrino events in coincidence with it. The deployment of such detectors in the ice is much easier than the IceCube DOMs because they are much closer to the surface on the ice. There are also a couple of test radio detectors deployed with the Auger observatory. Radio signals detectors could replace the fluorescent detectors in the future and increase the active time of the hybrid air shower arrays by a large factor. With the development of such new techniques we hope that the effective size of all detectors will increase and this will have a positive effect on size of all event samples.

Acknowledgments I am grateful to many friends and especially to Dave Seckel for the long discussions of these detection. My work on atmospheric neutrinos and on the arrival directions of different types of events is funded by DOE grant DE-FG02-91ER40626.

\section{References}

[1] Eli Waxman, John N. Bahcall, Phys.Rev. D59 (1999) 023002

[2] www.tevcat.uchicage.ecu

[3] R. Abbasi et al. (IceCube Collaboration), Phys. Rev. D84:072001 (2011)

[4] G.D. Barr et al., Phys. ReV. D70:023006 (2004)

[5] M.G. Aartsen et al. (IceCube Collaboration), Phys. Rev. Lett., 111:021103 (2013)

[6] M.G. Aartsen et al. (IceCube Collaboration), Science, 342:1242856 (2013)

[7] M.G. Aartsen et al. (IceCube Collaboration), Phys.Rev.Lett. 113:101101 (2014)

[8] G.A. Askaryan, JETP 14:441 (1962)

[9] R. Abbasi et al. (IceCube Collaboration), Nature 484, 351 (2012) 


\section{DISCUSSION}

VLADIMIR LIPUNOV: Is there any coincidence with GRB?

TODOR STANEV: IceCube has specifically looked for high energy neutrinos within a time limit of known gamma ray bursts. No such neutrinos were identified. This publication is [9] (R. Abbasi et al. (IceCube Collaboration), Nature 484, 351 (2012)) 